\title{
Bispecific antibodies and their use in applied research
}

\author{
Harshit Verma, Durairajan Ramachandran, Ashabaree Samal
}

Division of Biological Standardization

Indian Veterinary Research Institute, I zatnagar-243 122, India

Corresponding author: Harshit Verma, email: dr_harshitverma@yahoo.co.in

Received: 21-04-2012, Accepted: 26-05-2012, Published Online: 24-10-2012

doi: $10.5455 /$ vetworld.2012.775-780

\begin{abstract}
Bispecific antibodies (BsAb) can, by virtue of combining two binding specificities, improve the selectivity and efficacy of antibody-based treatment of human disease. Antibodies with two distinct binding specificities have great potential for a wide range of clinical applications as targeting agents for in vitro and in vivo immunodiagnosis, therapy and for improving immunoassays. They have shown great promise for targeting cytotoxic effector cells, delivering radionuclides, toxins or cytotoxic drugs to specific targets, particularly tumour cells. The development of BsAb research goes through three main stages: chemical cross linking of murine-derived monoclonal antibody, hybrid hybridomas and engineered BsAb. This article is providing the potential applications of bispecific antibodies.
\end{abstract}

Keywords: bispecific antibodies, immunoassay, immunotherapy, monoclonal antibody

\section{To cite this article:}

Verma H, Ramachandran D, Samal A (2012) Bispecific antibodies and their use in applied research, Vet World, 5(12): 775-780, doi: 10.5455/vetworld.2012.775-780

\section{Brief History}

In the first decade of this century Paul Ehrlich proposed the idea of using 'bodies' which possess a particular affinity for a certain organ [1]. The introduction of hybridoma technology for producing monoclonal antibodies [2] has revolutionized almost every field of modern medicine, including tumour targeting. Monoclonal antibodies seem to be the ideal 'magic bullets' for specific targeting of tumours.

\section{What is Antibody?}

Antibodies (also known as immunoglobulins, abbreviated Ig) are gamma globulin proteins that are found in blood or other bodily fluids of vertebrates, and are used by the immune system to identify and neutralize foreign objects, such as and viruses. They are typically made of basic structural units - each with two large heavy chains and two small light chains - to form a "Y" shaped molecule. This variable region, composed of 110-130 amino acids, give the antibody its specificity for binding antigen. The variable region includes the ends of the light and heavy chains.

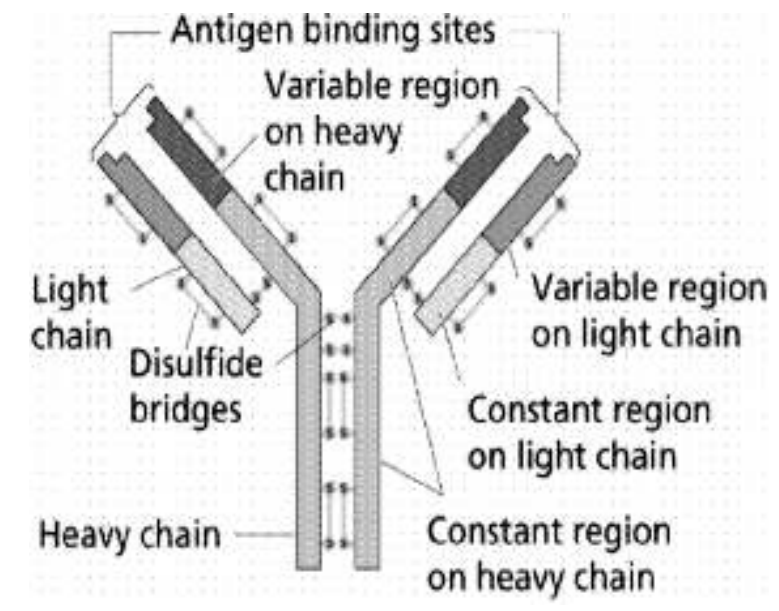

\section{Bispecific Antibody}

The potential for using monoclonal antibodies (MAb) to enhance the capacity of the immune system to combat tumors and infectious disease has 
stimulated imagination for the last 25 years [3]. In order to improve the therapeutic utility of antibodies different modifications have been suggested. One of these concerns the generation of BsAb [4]. Harmsen [5] developed an immunotherapy using Bispecific antibody that confers rapid protection against FMD in outbreak situations.

Immunotherapy of non-Hodgkin's B cell lymphoma with bispecific antibody construct has the potential to engage all cytotoxic T cells [6]. Bispecific monoclonal antibody (BsMAb), first introduced by Nisonoff and Rivers into the academia 40 years ago, is a unique type of MAb with two different binding specificities within a single molecule. Bispecific antibodies which have two different antigen-specific binding sites, one for tumour-associated antigen (target binding arm) and the other for the effector compounds (effector binding arm), have been developed. They can be used for any application in which two molecules need to be juxtaposed within a distance of a few nM.

BsMAb holds great promise for numerous therapeutic needs in the light of:

1. Recent breakthroughs in recombinant DNA technology,

2. The increased number of identified disease targets as the result of the completion of human genomic map project, and

3. A better understanding of the mechanism of human immune system.

A bsAb promotes the formation of conjugates between effectors and target cells by binding to a surface molecule on each cell type. If total random association of heavy and light chains occurs, ten different combinations of immunoglobulin molecules are generated; however, only one has the desired bispecific activity (Fig.-1). Fusions have been done between pairs of hybridoma cell lines secreting two existing monoclonal antibodies [7].

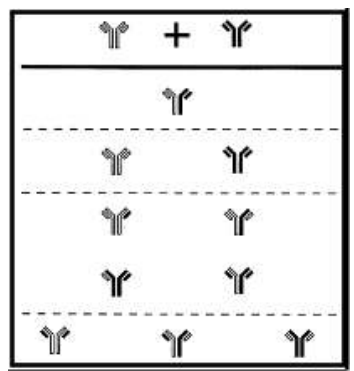

Fig.1 Diagrammatic representation of immunoglobulin molecules secreted by a hybrid hybridomas cell as a result of random association of heavy and light chain from both parental hybridomas.

\section{Applications of bispecific antibodies}

1) Immunoassay

2) Tumor targeting

3) Clinical application in cancer

4) Immunotherapy

5) Radioimmunotherapy

6) Drug/toxin/cytokine targeting

1) I mmunoassays: The effector binding arm can be designed to have specificity for marker enzymes or other indicator systems. The anti-target-anti-peroxidase bispecific antibodies which have been used in immunohistochemistry have led to improvements in sensitivity, signal-to-noise ratio, and simplification of staining procedures with preservation of fine ultra structural detail.

2) Tumour targeting: The use of bispecific antibodies for immunodiagnosis and therapy has shown some encouraging results. They have been used for delivering effector substances such as toxins and cytotoxic drugs to tumours, and some are now in clinical trials.

3) Clinical application in cancer: Immunotherapy is a powerful anti-cancer treatment modality. Using BsAbs, it is possible to take advantage of the highly specific binding characteristics of antibodies and combine these with the powerful effector functions of cytotoxic immune effector cells. BsAbs share two different, monoclonal antibody-derived, antigenrecognizing moieties within one molecule. By dual binding, BsAbs reactive with a trigger molecule on an immune effector cell on the one hand and a surface antigen on a tumor target cell on the other are thus able to functionally focus the lytic activity of the immune effector cell towards the target cell [8].

4) Radioimmunotherapy: Conventional radioimmunotherapy (RIT) using systemically administered MAb linked to radionuclides is a promising approach to metastatic cancer treatment. However, conventional RIT with MAb conjugates damages critical organs due to exposure to high radiation dose from long circulating radiolabeled antibody and non-specific accumulation of radiolabeled antibody in exposed organs. Pretargeted radioimmunotherapy with $\mathrm{BsMAb}$ is a multi-step strategy that allows quick and specific delivery of radioisotope (Y, I, Re and P) to a tumor with minimal radiation exposure, hence lowered toxicity to normal organs.

Pretargeting RIT combines long-circulating BsMAb and small, rapidly-excreted molecules to achieve a high tumor-to-blood ratio. The pretargeting 
approach allows the control of hematological toxicity, which is dose-limiting toxicity with conventional RIT.

5) I mmunotherapy: BsMAb is able to activate and target the cellular immune system to kill tumor cells or other pathogens. A number of different effector cells have been studied in this application like CTL $(\mathrm{CD} 8+)$ cells, natural killer cells, macrophages and polymorphonuclear cells. Two possible mechanisms have been proposed. First, CTL cells can express death ligands on their surfaces that can bind to the death receptors on the target cells, thereby initiating the target cell's apoptosis. Second, CTL cells can release perforins and granzymes in the narrow intercellular space between CTLs and target cells. Granzymes can enter the target cell to induce apoptosis of the target through the pores, which are created by perforins on the target cell membrane.

6) Drug/ toxin/ cytokine targeting: An expanding field in MAb-based therapeutics is the use of MAbs to direct selective cytotoxic agents. Chemical drugs, toxins and cytokines have all been conjugated to MAbs and are at various stages of development in clinical trials. BsMAb with intrinsic binding sites to any two antigens has the capability to form homogeneous and reproducible immunoconjugates. BsMAb has been constructed to deliver various drugs, e.g. doxorubicin, epirubicin, methotrexate or vinca alkaloids. In 2001 Ford [9] has successfully demonstrated a BsMAb targeting of doxorubicin to colon cancer cells expressing carcinoembryonic antigen (CEA) in vitro and in vivo. BsMAb has been expanded to target toxins, e.g. ricin A, saporin and gelonin to tumors. The advantage of using BsMAb to deliver high molecular weight toxins or drugs is that it avoids the complex chemistry involved in directly linking the antibody to the cytotoxic agent.

\section{Production of bispecific antibody (BsAb)}

$\mathrm{BsAb}$ are hybrid proteins that can be produced by chemical, genetic or biological methods. Bispecific antibodies are produced mainly by two methods:

1. Fusion of two different hybridoma cell lines

2. Chemically linking two antibody molecules

\section{Fusion of hybridomas}

* Normal plasma cells secrete only one set of heavy and light chains of immunoglobulin.

* Hybridoma cell lines producing monoclonal antibodies derived from the fusion of the 'nonsecreting' parental myeloma cell lines with immune cells.

* Fusion of two myeloma clones, each secreting different immunoglobulins, resulted in the codominant expression of both parental immunoglobulin genes and two sets of heavy and light chain were found to be secreted.

* Fusions have been done between pairs of hybridoma cell lines secreting two existing monoclonal antibodies.

* The main advantage of fusion technique is that the resulting bispecific antibodies are synthesized, assembled, and secreted by the same process as that of the native immunoglobulin.

* Once the 'hybrid hybridoma' cell lines are obtained, they will serve as the machines to produce endless amounts of antibody in the same way as normal hybridomas.

There are some disadvantages:

1. Difficulty of fusing hybrid hybridoma cells

2. Stability of the resulting cell lines

3. Low yields

4. Difficulty in purification of the bispecific molecules.

5. Cell fusion is labour-intensive, time consuming, and may not always succeed with the hybridoma pairs of choice.

6. Not all hybridoma cell lines exhibit good fusion performance.

7. Most parental hybridomas are derived from the fusion of HAT-sensitive myeloma fusion partners and immune spleen cells.

\section{Chemical linking of antibody molecules}

* The technique for producing bispecific antibodies by chemical manipulation was pioneered by Nisonoff \& Rivers.

* It doesn't require cell fusion.

* Chemical coupling can be achieved in two ways: direct coupling of the whole antibody molecules or their derivatives, and dissociation and reassociation of heterologous immunoglobulin.

* The latter requires chemical manipulation to dissociate immunoglobulins into half molecules without damaging the antigen binding site, then to reform the disulfide bonds linking heavy chains without allowing any interfering side reactions such as the formation of intrachain or mismatch disulfide bonds.

\section{Application of Bispecific Antibody}

1. Bispecific antibodies were synthesized by chemically cross linking bovine neutrophil monoclonal antibodies to $S$. aureus 305 capsule polysaccharide monoclonal antibodies [10]. To determine the 
influence of bispecific antibodies on neutrophil function, $S$. aureus 305 was preincubated with various concentrations of bispecific antibodies and neutrophils were then added to the opsonized bacteria at different bacteria to neutrophil ratios. The results indicated that bispecific antibodies that recognize both S. aureus 305 capsular polysaccharide and neutrophil antigens potentiate the bactericidal activity of neutrophils.

2. Bispecific antibodies are manufactured or engineered from two separate antibodies to create a molecule with two different binding specificities. These are used to link a target to trigger molecules on effector cells of the immune system. This shows outlines the preparation of bispecific antibodies, describes the components of cellular cytotoxicity in the immune system [11].

3. The use of bispecific antibodies as possible therapeutic agents for cancer treatment was proposed in the mid-1980s [12]. The common strategy for making bispecific antibodies involves combining the variable domains of the desired mAbs into a single bispecific structure. Many different formats of bispecific antibodies have been generated within the research field of bispecific immunotherapeutics, including the chemical heteroconjugation of two complete molecules or fragments of MAbs, quadromas, $\mathrm{F}\left(\mathrm{ab}^{\prime}\right) 2$, diabodies, tandem diabodies and single-chain antibodies.

4. Jackman and their co workers [13] had applied a technology to generate a bispecific antibody suitable for development as a human therapeutic. This antibody directly inhibits the activation of the high affinity IgE receptor Fc $\gamma \mathrm{RI}$ on mast cells and basophils by cross-linking FcyRI with the inhibitory receptor FcyRIIb, an approach that has strong therapeutic potential for asthma and other allergic diseases.

5. In 2010 Wang and their coworker report that the natural bispecific antibody can also be generated in New Zealand white rabbits by immunization with synthesized conjugates. These antibodies showed bispecificity to the components that were simultaneously used to immunize the animals. Wang observed a trend in test animals that female rabbits exhibited stronger bispecific antibody responses than males. The bispecific antibody was monomeric and primarily belonged to immunoglobulin (Ig) G. Moreover, bispecific antibodies were demonstrated by mixing 2 purified monospecific antibodies in vivo and in vitro [14].

6. In 2010 Kammila and their co workers worked for the detection of severe acute respiratory syndrome
(SARS) a simple, easy to use immunoswab method was developed by generating a panel of monoclonal antibodies (MAbs), Bispecific MAbs and chicken polyclonal IgY antibody against the SARS-CoV nucleocapsid protein (NP). The key feature of this simple immunoswab diagnostic assay is its ability to detect the presence of the SARS-CoV antigen within 45-60 min with the availability of the body fluid samples [15].

7. In 2011 Schaefer correctly assemble two heavy and two light chains, derived from two existing antibodies, to form human bivalent bispecific IgG antibodies without use of artificial linkers. Applying the three possible "CrossMab" formats, they generated bispecific antibodies against angiopoietin-2 (Ang-2) and vascular endothelial growth factor A (VEGF-A) and show that they can be produced by standard techniques, exhibit stabilities comparable to natural antibodies, and bind both targets simultaneously with unaltered affinity. Because of its superior sideproduct profile, the CrossMab ${ }^{\mathrm{CH}-\mathrm{CL}}$ was selected for in vivo profiling and showed potent antiangiogenic and antitumoral activity [16].

8. In 2010 Robert describes a new procedure for forming multivalent and/or multispecific proteins, known as the dock-and-lock (DNL) technique. This methodology has the flexibility to create a number of other biologic agents of therapeutic interest. A variety of constructs, based on anti-CD20 and CD22 antibodies, have been made, with results showing that multispecific antibodies have very different properties from the respective parental monospecific antibodies. Thus, DNL technology is a highly flexible platform that can be used to prepare many different types of agents that could further improve cancer detection and therapy [17].

9. In 2011 Schanzer described novel tetravalent, bispecific antibody derivatives that bind two different epitopes on the HIV coreceptor CCR5. In contrast to monospecific CCR5 antibodies, bispecific antibody derivatives block two alternative docking sites of CCR5-tropic HIV strains on the CCR5 coreceptor. Consequently, these molecules showed 18- to 57-fold increased antiviral activities compared to the parent antibodies. Most importantly, one prototypic tetravalent CCR5 antibody had antiviral activity against virus strains resistant to the single parental antibodies. In summary, physical linkage of two CCR5 antibodies targeting different epitopes on the HIV coreceptor CCR5 resulted in tetravalent, bispecific antibodies with enhanced antiviral potency against wild-type and CCR5 antibody-resistant HIV-1 strains [18]. 
Bispecific antibodies and their use in applied research

Table.1. Qualities of bispecific and monoclonal antibodies

\begin{tabular}{lll}
\hline S. No. & Bispecific Antibody (BsAb) & Monoclonal antibody (Mab) \\
\hline 1. & Design flexibility, various BsAb formats & Excellent pharmacokinetics; long half life \\
2. & More specific and efficient targeting & Straightforward production/purification \\
3. & Optimal selectivity for activator/down-regulatory molecules & Optimal stability in-vivo \\
4. & High affinity interactions: effector cells can be "armed" BsAb & \\
5. & Better tissue peneteration & \\
\hline
\end{tabular}

10. In 2011 wang and jinming identified the presence of natural bispecific antibodies against cyclic citrullinated peptide (CCP) and immunoglobulin $\mathrm{G}$ (IgG) in RA patients' sera by means of a doubleantigen sandwich enzyme-linked immunosorbent assay (ELISA). The spontaneous emergence of bispecific antibodies was confirmed by mixing different proportions of 1 anti-CCP-positive serum and 1 rheumatoid factor (RF)-positive serum in vitro and conclude that the natural occurrence of bispecific antibodies in human diseases may provide new insights for a better understanding of the diseases [19].

\section{Conclusion}

With its unique two-arm structures, bispecific monoclonal antibody provides powerful tools for targeted-delivery drugs, toxins, cytokines, enzymes, DNAs and radionuclides. Besides, BsMAb is able to activate the immune defense system by artificially combining humoral and cellular components to retarget them to tumors or viral infected cells. BsAb are able to trigger a plethora of biological responses, making them attractive candidates for immunotherapy. The nature of both the trigger molecule and the target antigen will determine the efficacy of BsAb-based treatments.

\section{Future perspectives}

Bispecific antibody possesses a number of beneficial characteristics, some of which overcome the limitations of Monoclonal antibodies. The distinct pharmacokinetics of BsAb, however, requires them to be used in a different way than MAbs. Adoptive cellular therapy, using effector cells 'armed' with $\mathrm{Bs} \mathrm{Ab}$, might significantly improve the therapeutic utility of $\mathrm{BsAb}$ and increased understanding of the underlying mechanisms of successful antibody-based therapies.

The newly identified Toll-like receptor (TLR) family are implicated in innate host defense against bacteria. TLR-2 and TLR-4 recognize bacterial cell wall components in vivo, making these receptors potential candidates for BsAb. Finally, the recent 'genomic revolution' has increased the number of potential therapeutic target antigens from $<400$ to $<4,000$. We anticipate that BsAb will play a significant role in therapies directed to some of these novel targets.

\section{References}

1. Ehrlich, P. (1906). Collected Studies on Immunity John Wiley, New York. 2: 442.

2. Kohler, G. and Milstein, C. (1975) Continuous cultures of fused cells secreting antibody of predefined specificity, Nature., 256:495-497.

3. Weiner, L.M. (1999) An overview of monoclonal antibody therapy of cancer. Semin. Oncol., 26:41-50.

4. Van de Winkel, J.G.J. (1997) Immunotherapeutic potential of bispecific antibodies. Immunol. Today., 18: 562-564.

5. Harmsen, M. M., Fijten, H. P. D., Dekker, A. and Eble, P. L. (2008) Passive immunization of pigs with bispecific llama single-domain antibody fragments against foot-and-mouth disease and porcine immunoglobulin. Veterinary Microbiology., 132 (1/2): 56-64.

6. Larski, Z. (2009) Some new data concerning virology and immunology. Medycyna Weterynaryjna., 65(4): 219-222.

7. Songsivilai, S. and Lachmann, P. J. (1990) Bispecific antibody: a tool for diagnosis and treatment of disease. Clin. exp. Immunol., 79:315-321.

8. Kroesen, B.J., Helfrich, W., Molema, G. and Leij, L. de. (1998) Bispecific antibodies for treatment of cancer in experimental animal models and man. Advanced Drug Delivery Reviews., 31:105-129.

9. Ford, C.H., Osborne, P.A., Rego, B.G., Mathew, A. (2001) Bispecific antibody targeting of doxorubicin to carcinoembryonic antigen-expressing colon cancer cell lines invitro and invivo. Int. J. Cancer., 2: 851-855.

10. Tomita, G.M., Wang, Y., Paape, M.J., Poultrel, B. and Rainard, P. (2000) Influence of bispecific antibodies on the in vitro bactericidal activity of bovine neutrophils against Staphylococcus aureus. Journal ofDairy Science., 83(10): 2269-2275.

11. Fanger, M.W. (1995) Bispecific antibodies, R.G. Landes Publishers.

12. Thakur, A. and Lum, L.G. (2010) Cancer therapy with bispecific antibodies: Clinical experience. Curr Opin MolTher., 12(3):340-9.

13. Janet, Jackman., Yongmei, Chen., Arthur Huang, 
Barbara Moffat., Justin M. Scheer, Steven R. Leong., Wyne P. Lee., Juan Zhang. and Lawren, C. Wu. (2010) Development of a Two-part Strategy to Identify a Therapeutic Human Bispecific Antibody That Inhibits IgE Receptor Signaling. J Biol Chem., 285(27): 20850-20859.

14. Wang, W., Ruihuan Xu and Jinming Li. (2010) Production of Native Bispecific Antibodies in Rabbits. PLoS One., 5(6):10879.

15. Kammila, S., Dipankar Das, Bhatnagar, P. K., Sunwoo, H.H., Zayas Zamora, G., King, M. and Suresh, M.R. (2010) A rapid point of care immunoswab assay for SARS-CoV detection. Journal of Virological Methods., 152(1/2): 77-84.

16. Wolfgang Schaefer, Jörg T. Regula, Monika Bähner, Jürgen Schanzer, Rebecca Croasdale, Harald Dürr, Christian Gassner, Guy Georges, Hubert Kettenberger, and Christian Klein. (2011) Immunoglobulin domain crossover as a generic approach for the production of bispecific IgG antibodies. Proc NatlAcad Sci U S A., 108(27): 11187-11192.

17. Robert, M. Sharkey, , Chien-Hsing Chang, and David M. Goldenberg. (2010) Improved Cancer Therapy and Molecular Imaging with Multivalent, Multispecific Antibodies. Cancer Biother Radiopharm., 25(1): 1-12.

18. Jürgen Schanzer, Andreas Jekle, , Adriane Lochner, Rebecca Croasdale, Marianna Dioszegi, Jun Zhang, Eike Hoffmann, Michael Brandt, Pablo Umana and Ulrich Brinkmann. (2011) Development of Tetravalent, Bispecific CCR5 Antibodies with Antiviral Activity against CCR5 Monoclonal Antibody-Resistant HIV-1 Strains. Antimicrob Agents Chemother., 55(5): 2369-2378.

19. Wei Wang and Jinming Li. (2011) Identification of Natural Bispecific Antibodies against Cyclic Citrullinated Peptide and Immunoglobulin $G$ in Rheumatoid Arthritis. PLoS One., 6(1): e16527. 